\title{
Advanced Nanofabrication using Helium, Neon and Gallium Ion Beams in the Carl Zeiss Orion NanoFab Microscope
}

\author{
Danielle Elswick, Mohan Ananth, Lewis Stern, Jeff Marshman, Dave Ferranti and Chuong Huynh \\ Carl Zeiss Microscopy, Ion Microscopy Innovation Center, Peabody, MA USA
}

Direct write focused ion beam (FIB) machining represents the fastest and most flexible method to fabricate nano-scaled devices for prototyping and research applications. The use of a FIB combined with SEM allows for immediate inspection and refinement steps in the patterning process to assure the desired fidelity. FIB technology has thus found wide-spread use in fields such as photonics, nanofluidics, TEM sample preparation, integrated circuit modification, MEMS and more. Conventional gallium FIBs use a liquid metal ion source (LMIS) with many notable drawbacks such as a lower limit for feature sizes that can be achieved and undesirable Ga implantation. Ion microscopy with helium and neon beams created from a gas field ion source (GFIS) demonstrates great flexibility for many nanofabrication applications. The beam-sample interaction dynamics of helium and neon beams at moderate voltages extends direct write fabrication and inspection further into the sub-10 nm regime. The Carl Zeiss Orion NanoFab is a commercially available ion beam microscope offering three different species of ions to extend beyond the limits of gallium with the addition of helium and neon beams.

Nanophotonic and plasmonic devices with $5 \mathrm{~nm}$ machining precision and nearly vertical sidewalls have been fabricated using GFIS technology. An example of machining over multiple length scales is given in Figure 1 which shows a double bowtie plasmonic device inspired by the work of Zhang [1] and coworkers. A three step approach was used to fabricate this double bowtie structure in a $100 \mathrm{~nm}$ thick gold film on a glass substrate. Ga FIB was used during the first step to open up a $1 \mu \mathrm{m}$ square window into the film leaving behind a square island $200 \mathrm{~nm}$ wide. The second step consisted of using the Ne FIB to machine slits in the island which then formed a cross. Sputtering with Ne also avoids Ga contamination into the critical area around the bowtie. Finally, He ion milling was utilized to separate the four triangular elements with a $5 \mathrm{~nm}$ radius of curvature of the vertices. This produced a final structure where the active area of the device had never been exposed to the Ga beam. Figure 2 is another example of a plasmonic device machined in $100 \mathrm{~nm}$ thick Au using the He beam. Helium offers fine precision with beam control to enable patterning of a $4 \mathrm{~nm}$ gap between the two Au antennae.

Several other popular applications using $\mathrm{He}$ and $\mathrm{Ne}$ ion beams include nanopores, graphene patterning, lithography and ion beam induced deposition for applications such as circuit edit. Helium has been used to create nanopores down to $3 \mathrm{~nm}$ in diameter, with aspect ratios greater than 10:1, in a variety of materials for DNA sequencing applications. The process takes only seconds, is repeatable and scalable. Initial detection of DNA translocation events have been demonstrated [2]. The band gap of graphene can be modulated by reducing the width of patterned graphene nanoribbons. The He beam can easily machine 5-6 nm wide graphene nanoribbons without damage. Direct write lithography in commercial resists HSQ and PMMA produced line features down to $4 \mathrm{~nm}$ in width. There is greatly reduced proximity effects using $\mathrm{He}$ and $\mathrm{Ne}$ ions as compared to electron beam exposure, so dense and sparse patterns develop identically with no proximity effects correction necessary. The resist sensitivity is an order of magnitude higher for helium exposure as compared to electrons [3] and increases even further with neon exposure. And finally, the use of $\mathrm{He}$ and $\mathrm{Ne}$ ion beams for circuit edit applications is being developed. Deposition of conducting and insulating materials using $\mathrm{He}$ and $\mathrm{Ne}$ create features smaller 
than what is possible with Ga FIB and with no observed proximity effects [4]. New Ne induced beam chemistry is being developed for low resistivity metal deposition and also via formation by ion milling. Some examples are provided in Figure 3.

The Orion NanoFab and a gallery of nanofabrication will be presented showcasing the capability and performance of this novel microscope. Features size and material removal via conventional gallium FIB systems is now surpassed using neon and helium ion beams. The technology behind Orion NanoFab has touched a wide array of applications that range from nanomachining $5 \mathrm{~nm}$ pores for single molecule detection to patterning devices in graphene and creating nanophotonic devices in thin films.

\section{References:}

[1] Z. Zhang, et al., Nano Letters, 9, 4505 (2009).

[2] Yang, et al., Nanotechnology 22, p.285310 (2011).

[3] Winston et al., JVST B 27 (6), p.2702 (2009).

[4] Scipioni et al., Microscopy Today, 19 (3), p.22 (2011).

[5] Figure 2. Dr. Nestor Zaluzec who is thanked for his collaboration and TEM work.
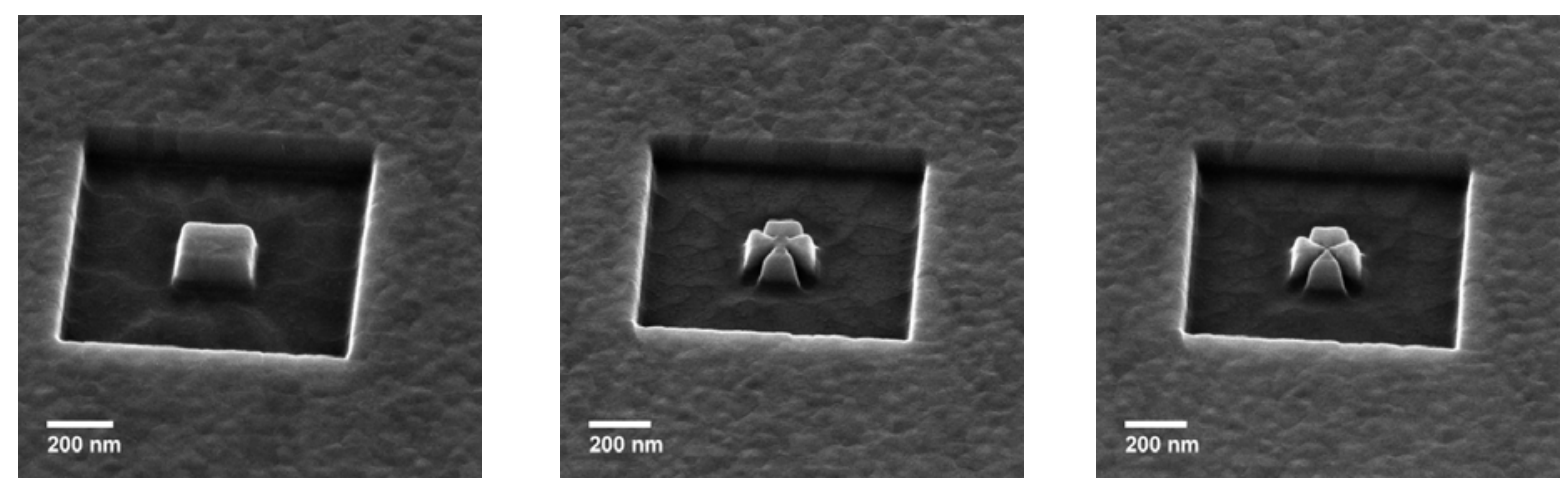

Figure 1: Plasmonic device (100 nm thick gold film on glass ) machined in three steps using $\mathrm{Ga}, \mathrm{Ne}$ and He beams respectively from left to right.

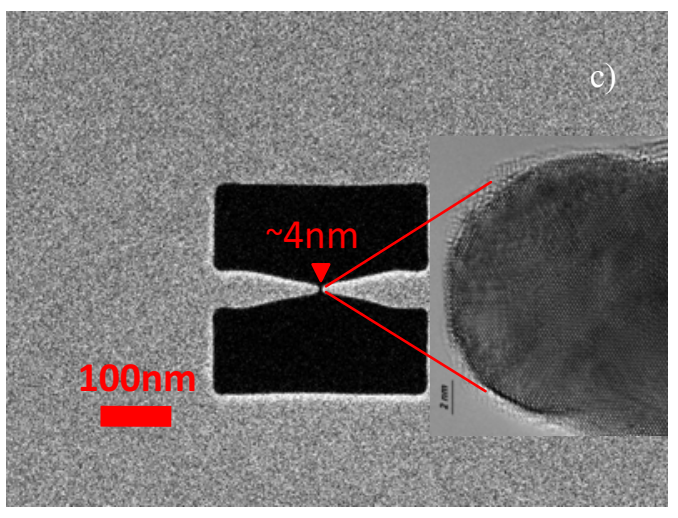

Figure 2: Two gold antennae separated by $4 \mathrm{~nm}$, machined in $100 \mathrm{~nm}$ thick gold using He ion beam. Inset shows TEM of gold tip [5].
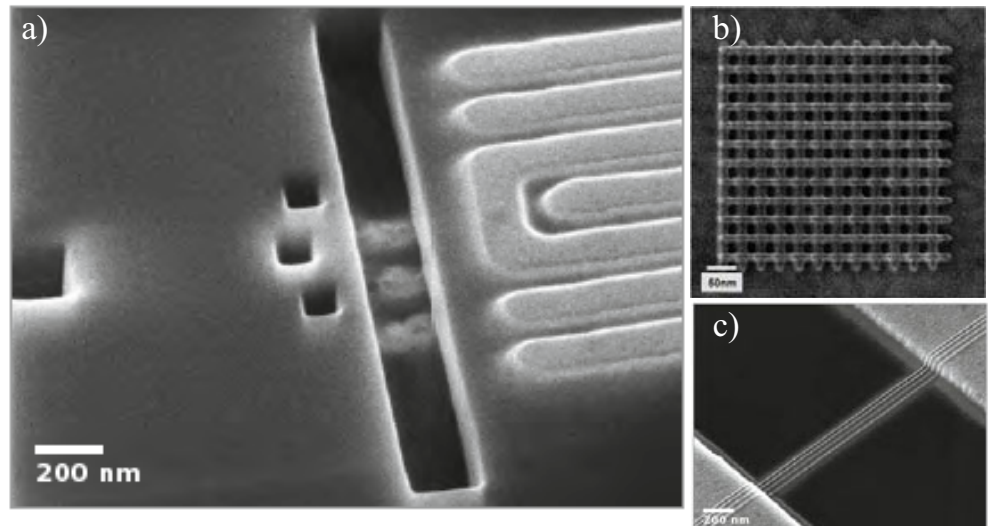

Figure 3: a) Rectangular hole milled through $\sim 300 \mathrm{~nm}$ of dielectic using neon beam. b) $15 \mathrm{~nm}$ insulators with $10^{11} \mathrm{ohm} . \mathrm{cm}$ resistivity. c) $10 \mathrm{~nm}$ metal lines with resistivity of $<200 \mu \mathrm{ohm} . \mathrm{cm}$. 